\title{
Bibliography: Studies and Editions of Isaac Orobio de Castro
}

\section{Specialised Studies on Orobio}

Benítez, Miguel. "Orobio de Castro et la littérature clandestine." XVIII siècle 14 (1982): 219-226. Reprinted in La face cachée des Lumières: Recherches sur les manuscrits philosophiques clandestins de l'âge classique, by Miguel Benítez, 147-154. Paris: Universitas, 1996.

Caballero, Práxedes. "La crítica de Orobio de Castro a Spinoza." In Spinoza y España, edited by Atilano Domínguez, 229-237. Cuenca: Ediciones de la Universidad de Castilla-La Mancha, 1994.

Carvalho, Joaquim de. Oróbio de Castro e o espinosismo. Lisboa: Seara Nova, 1940.

Cohen, Robert. "Yosef Kaplan, Isaac Orobio de Castro, and the Sephardi World of Seventeenth-Century Amsterdam." Studia Rosenthaliana 23, no. 2 (1989): 186-190.

Díaz Esteban, Fernando. "Entre la religión y la filosofía: La polémica de Isaac Orobio y Alonso de Zépeda." Sefarad 61, no. 2 (2001): 319-344; 62, no. 1 (2002): 21-55.

Dupuy, Bernard. "Deux contemporains de Spinoza: Abraham Pereyra et Isaac Orobio de Castro." Istina 38 (1993): 287-304.

Esaguy, Augusto d'. "The Dramatic Life of Orobio de Castro." Bulletin of the Institute of the History of Medicine 5.9 (November 1937): 822-826.

Feldman, Seymour. "Ha-bikoret hayehudit ha-rishonah neged Shpinozah.” Iyyun 37, no. 3-4 (1988): $222-237$.

Friedenwald, Harry. Jewish Luminaries in Medical History and a Catalogue of Works Bearing on the Subject of the Jews and Medicine from the Private Library of Harry Friedenwald. Baltimore: Johns Hopkins University Press, 1946, 53-56.

Gebhardt, Carl. “Juan de Prado." Chronicon Spinozanum 3 (1923): 269-291.

Glatzer, Michael. "Mi-natsrut biSefarad leyahadut beHoland.” Peamim 15 (1983): 137-141.

Graetz, Heinrich. "Don Balthasar Isaak Orobio de Castro: Eine biographische Skizze." Monatsschrift für die Geschichte und Wissenschaft des Judenthums 16 (1867): 321-330.

Graetz, Heinrich. Geschichte der Juden von den ältesten Zeiten bis auf die Gegenwart, vol. 10: Geschichte der Juden von der dauernden Ansiedelung der Marranen in Holland (1618) bis zum Beginn der Mendelssohnischen Zeit (1760). Leipzig: Leiner, 1868.

Israel, Jonathan I. "Orobio de Castro and the Early Enlightenment." In Mémorial I.-S. Révah: Études sur le marranisme, l'hétérodoxie juive et Spinoza, edited by Henry Méchoulan and Gérard Nahon, 227-245. Paris: Peeters, 2001.

Julius, Rafael. “Doktor Yitsḥak, ha-anus she-ḥazar leyahadut.” Maariv 5 (August 1983): 38.

Kaplan, Yosef. “El testimonio de Baltasar Álvarez de Orobio sobre la peste en Málaga en 1637," Helmantica 29/89 (1978): 212-232.

Kaplan, Yosef. Yitshak Orobio de Kastro uvenei hugo. PhD dissertation, The Hebrew University, 1978.

Kaplan, Yosef. "Yitsḥak Orobio de Kastro: Darko shel anus min ha-natsrut la-yahadut." Peamim 5 (1980): $29-38$.

Kaplan, Yosef. Mi-natsrut leyahadut: Hayyaw ufe'alo shel ha-anus Yitsḥak Orobio de Kastro. Jerusalem: Magnes Press, 1982.

Kaplan, Yosef. From Christianity to Judaism. The Story of Isaac Orobio de Castro. Oxford: Oxford University Press, 1989. 
Kaplan, Yosef. "Isaac Orobio de Castro." In Die Philosophie des 17. Jahrhunderts, Band 2: Frankreich und Niederlande, ed. Jean-Pierre Schobinger, 889-891, 973. Basel: Schwabe \& Co., 1993.

Kayserling, Meyer. Biblioteca española-portugueza-judaica: Dictionnaire bibliographique. Strassburg: Trübner, 1890, 81-83.

Nahon, Gérard. "Échec à l'Inquisition de Séville: Isaac Orobio de Castro (1620-1687).” Yod 26 (1989): 57-62.

Polak, Leo. "De betekenis der Joden voor de wijsbegeerte," in Geschiedenis der Joden in Nederland, Eerste deel (tot circa 1795), ed. Hendrik Brugmans and Abraham Frank, 680-713. Amsterdam: Van Holkema \& Warendorf, 1940.

Popkin, Richard H. "Orobio de Castro, Isaac," Encyclopaedia Judaica, vol. XII, 1475-1477. Jerusalem: Keter, 1972, reimpressed in Berenbaum, Michael and Fred Skolnik, ed. Encyclopaedia Judaica, 2nd. ed., vol. XV, 481-482. Detroit: Macmillan Reference USA, 2007.

Popkin, Richard H. "Jewish Anti-Christian Arguments as a Source of Irreligion from the Seventeenth to the Early Nineteenth Century." In Atheism from the Reformation to the Enlightenment, edited by Michael Hunter and David Wootton, 159-181. Oxford: Clarendon Press, 1992.

Rensoli Laliga, Lourdes. "Isaac Orobio de Castro: Intellectual Marrano, Intellectual Jew." Journal of Spanish, Portuguese, and Italian Crypto Jews 3 (2011): 67-74.

Révah, I. S. Spinoza et le Dr Juan de Prado. Paris: Mouton \& Cie, 1959.

Révah, I. S. "Orobio de Castro et sa famille aux prises avec l'Inquisition espagnole." Bijdragen en Mededelingen van het Genootschap voor de Joodsche Wetenschap in Nederland 9 (1965): 87-90.

Révah, I. S. Des Marranes à Spinoza. Edited by Henry Méchoulan, Pierre-François Moreau and Carsten Lorenz Wilke. Paris: Vrin, 1995.

Rooden, Peter T. van and Jan W. Wesselius. "The Early Enlightenment and Judaism: the "Civil Dispute' between Philippus van Limborch and Isaac Orobio de Castro (1687)." Studia Rosenthaliana 21 (1987): 140-153.

Ruderman, David. "Yosef Kaplan, Mi-natsrut le-yahadut." Zion 49, no. 3 (1984): 306-313.

Salomon, Herman Prins. "Baruch Spinoza, Ishac Orobio de Castro and Haham Mosseh Refael de Aguilar on the Noahites: A Chapter in the History of Thought." Arquivos do Centro Cultural Português 14 (1979): 253-286.

Schoeps, Hans-Joachim. "Isaac Orobio de Castros Religionsdisput mit Philipp van Limborch." Judaica 2 (1946): 89-105.

Silva Rosa, Jacob S. da. "Van Marrano tot Joodsch Apologeet: Dr. Isaac (Balthazar) Orobio de Castro (omstr. 1620-1687).” De Vrijdagavond (27 March, 1926): 6-9; (28 March, 1926): 21-23.

Silvera, Myriam. "L'esilio del popolo ebraico nelle Prevenciones divinas di Isaac Orobio de Castro nel suo dialogo con Philip Van Limborch." In In the Iberian Peninsula and Beyond: A History of Jews and Muslims (15th-17th Centuries), vol. I, edited by José Alberto Rodrigues da Silva Tavim, Maria Filomena Lopes de Barros, and Lúcia Liba Mucznik, 328-347. Newcastle upon Tyne: Cambridge Scholars Publishing, 2015.

Silvera, Myriam. "D'Holbach and the Dissertation sur le Messie: Some Enigmas, and a New Source." In Portuguese Jews, New Christians, and "New Jews": A Tribute to Roberto Bachmann, ed. Claude B. Stuczynski and Bruno Feitler, 361-384. Leiden: Brill, 2018.

Sutcliffe, Adam. "Judaism and Jewish Arguments in the Clandestine Radical Enlightenment." In Scepticisme, clandestinité et libre pensée, edited by Gianni Paganini, Miguel Benítez and James Dybikowski, 97-113. Paris: Champion, 2002.

Sutcliffe, Adam. Judaism and Enlightenment. Cambridge: Cambridge University Press, 2003. 
Szapiro, Elie. "Un document sur le séjour à Toulouse d'Isaac Orobio de Castro." Revue des études juives 138, no. $3-4$ (1979): 385-389.

Taub, Emmanuel. "Mesianismo y ley: Frente al problema del Mesías en la tradición sefaradí de Maimónides e Isaac Oróbio de Castro." Sefárdica 19 (2010): 221-231.

Wilke, Carsten. "Isaak Orobio de Castro." In Metzler Lexikon jüdischer Philosophen: Philosophisches Denken des Judentums von der Antike bis zur Gegenwart, edited by Andreas B. Kilcher and Otfried Fraisse, 153-155. Stuttgart: Verlag J. B. Metzler, 2003.

\section{Printed Editions of Orobio's Works in Chronological Order}

Epílogo de lo que passó en la peste de la ciudad de Málaga este año de 1637 (Málaga: Juan Serrano de Vargas y Uruena, 1637). Edition in Kaplan, "El testimonio," 219-231; and Kaplan, From Christianity to Judaism, 387-401.

Controvertitur utrum materialibus morbis in choantibus sang. missio revulsiua, iuxta Hippocratis, \& Galeni dogmata per distantissimas venas effici debeat? Opus editum a Doctore $D$. Balthasare de Orobio, Excellentissimi D. Ducis de Medina Coeli, \& Alcala â cubiculo medico, quondam insignis Collegii Theologorum Cõplutensium collega, \& Hispalensis celebris Vniuersitatis methodi Cathedrae proprietario moderatore. (Seville: apud Ignatium de Lyra, 1653).

Pergunta que me fez o eminentisimo Senhor Doutor Orobio, by Mosseh Rephael d'Aguilar (manuscript). Edition in Salomon, "Baruch Spinoza, Ishac Orobio de Castro," 255-257.

Epístola invectiva contra un filósofo médico que dudaba o no creía la verdad de la Divina Escritura (manuscript, Winter 1663-1664). Abridged edition in Révah, Spinoza, 84-129.

Carta apologética del doctor Ishack Orobio de Castro al Doctor Prado (manuscript, 1664). Edition in Révah, Spinoza, 130-142.

Carta al hijo del doctor Prado (manuscript, dated from Weycke, August 12, 1664). Edition in Révah, Spinoza, 143-153.

Respuesta a una persona que dudava si el libro de Raymundo Lulio nuevamente traducido y comentado por don Alonso de Zepeda era inteligible y si concluyan sus discursos. Edition in: Alonso de Zepeda, Defensa de los términos y doctrina de Raymundo Lullio contra cierto rescribiente judío de la sinagoga de Amsterdam, Brussels, 1666, 2-77.

Explicación parafrástica del cap. 53 del profeta Isaías (manuscript). Edition in Adolf Neubauer, The Fifty-Third Chapter of Isaiah According to the Jewish Interpreters, vol. I. Oxford: J. Parker, 1876, 21-153; reprint New York 1969.

Respuesta a un escrito que presentó un predicante francés al autor contra la observancia de la divina Ley de Moseh (manuscript). Edition in Moses Bensabat Amzalak, La observancia de la Divina Ley de Mosseh, manuscrito do século XVII publicado por primera vez, com um estudo prévio. Coimbra: Imprensa da Universidade, 1925, 1-101, reprint Barcelona: Riopiedras, 1991.

Prevenciones divinas contra la vana idolatría de las gentes (manuscript). Edizione critica, con introduzione, note di commento e riassunti parafrasi in italiano a cura di Myriam Silvera, vol. I. Florence: L. S. Olschki, 2013.

Certamen philosophicum propugnatae veritatis divinae ac naturalis: Adversus J[oannes] $B$ [redenburgii] Principia, in fine annexa, ex quibus quod religio rationi repugnat demonstrare nititur, quo in Atheismi Spinosae baratro immersus jacet, quod religio nil rationi repugnans credendum proponit, evidenter ostenditur. Amsterdam: n.p., 1684. Reprint Amsterdam: Theodor Ossaan, 1703; and in François de Salignac de La Mothe Fénelon, Refutation des erreurs de Benoit de Spinosa. Brussels: Francois Foppens, 1731, 387-483.

Philippus van Limborch. De veritate religionis christianae amica collatio cum erudito Judaeo. Gouda: Apud Justum ab Hoeve, 1687. Reprint Farnborough: Gregg, 1969. 
Israël vengé, ou Exposition naturelle des prophéties hébraïques que les chrétiens appliquent à Jésus leur prétendu Messie, traduit sur le manuscrit par Henriquez. Edited by Baron d'Holbach. London: n.p., 1770. English translations: Israel Defended, or the Jewish Exposition of the Hebrew Prophecies Applied by the Christians to their Messiah. Translated from the French [by Grace Aguilar] and Printed Expressly for the Use of Young Persons of the Jewish Faith. London 1838; Israel Avenged. Translated and answered by the Rev. Alex. McCaul of Trinity College (Dublin and London, 1839-1840).

Entretiens sur divers sujets d'histoire et de religion entre Mylord Bolingbroke et Isaac d'Orobio, rabin des juifs portugais à Amsterdam. n.p., 1770 (a literary hoax inspired by Israël vengé, the text being a reprint of the Entretiens sur divers sujets d'histoire, de littérature, de religion et de critique that Mathurin Veyssière de La Croze had published anonymously in Rotterdam, indicating Cologne, in 1711).

\section{Digitised Manuscripts Accessible Online}

(For a full inventory of the manuscripts, see Kaplan, From Christianity to Judaism, 431-440)

Amsterdam, Ets Haim, ms. 48 A 12: http://etshaimmanuscripts.nl/manuscripts/eh-48-a-12/

Amsterdam, Ets Haim, ms. 48 A 21: http://etshaimmanuscripts.nl/manuscripts/eh-48-a-21/

Amsterdam, Ets Haim, ms. 48 A 23: http://etshaimmanuscripts.nl/manuscripts/eh-48-a-23/

Amsterdam, Ets Haim, ms. 48 B 6: http://etshaimmanuscripts.nl/manuscripts/eh-48-b-05/

Amsterdam, Ets Haim, ms. 48 B 12: http://etshaimmanuscripts.nl/manuscripts/eh-48-b-12/

Amsterdam, Ets Haim, ms. 48 C 1, 2: http://etshaimmanuscripts.nl/manuscripts/eh-48-c-01-02/

Amsterdam, Ets Haim, ms. 48 C 4: http://etshaimmanuscripts.nl/manuscripts/eh-48-c-04/

Amsterdam, Ets Haim, ms. 48 C 12: http://etshaimmanuscripts.nl/manuscripts/eh-48-c-12/

Amsterdam, Ets Haim, ms. 48 C 16: http://etshaimmanuscripts.nl/eh_48_c_16/

Amsterdam, Ets Haim, ms. 48 D 6: http://etshaimmanuscripts.nl/manuscripts/eh-48-d-06/

Amsterdam, Ets Haim, ms. 48 D 16: http://etshaimmanuscripts.nl/manuscripts/eh-48-d-16/

Amsterdam, Ets Haim, ms. 48 E 42: http://etshaimmanuscripts.nl/manuscripts/eh-48-e-42/

Amsterdam, Ets Haim, ms. 49 A 16: http://etshaimmanuscripts.nl/manuscripts/eh-49-a-16/

Bordeaux, Bibliothèque municipale, cod. 828: http://bibliotheque.bordeaux.fr/in/faces/details. xhtml?id=BordeauxS_B330636101_Ms828_033_005

Hamburg, Staats- und Universitätsbibliothek, hebr. 85a: http://digitalisate.sub.uni-hamburg.de/nc/ detail.html?id=1901\&tx_dlf\%5Bid\%5D=25088\&tx_dlf\%5Bpage\%5D=7

Hamburg, Staats- und Universitätsbibliothek, hebr. 240a: http://digitalisate.sub.uni-hamburg.de/ nc/detail.html?id=1901\&tx_dlf\%5Bid\%5D=24970\&tx_dlf\%5Bpage\%5D=5

Hamburg, Staats- und Universitätsbibliothek, Levy 122: http://digitalisate.sub.uni-hamburg.de/nc/ detail.html?id=1901\&tx_dlf\%5Bid\%5D=25061\&tx_dlf\%5Bpage $\% 5 \mathrm{D}=5$

Madrid, Biblioteca nacional, cod. 18.249: http://bdh.bne.es/bnesearch/detalle/bdh0000135633

New York, Jewish Theological Seminary, MS 2535: https://primo-tc-na01.hosted.exlibrisgroup.com/ primo-explore/fulldisplay?docid=JTS_DIGITOOL231960\&context=L\&vid=JTS\&search_scope= JTS\&tab=default_tab\&lang=en_US

New York, Jewish Theological Seminary, MS 2537: https://primo-tc-na01.hosted.exlibrisgroup.com/ primo-explore/fulldisplay?docid=JTS_DIGITOOL231958\&context=L\&vid=JTS\&search_scope= JTS\&tab=default_tab\&lang=en_US

Oxford, Bodleian Library, Opp. Add. $4^{\circ}$ 51: https://digital.bodleian.ox.ac.uk/inquire/p/60567506170c-4192-94e4-5db213e177b9

Oxford, Bodleian Library, Opp. Add. $4^{\circ} 147 a$ : https://digital.bodleian.ox.ac.uk/inquire/p/cccc39c83299-460d-9217-d724b0ec65d8 
Oxford, Bodleian Library, Opp. Add. $4^{\circ}$ 147b: https://digital.bodleian.ox.ac.uk/inquire/p/ b557972a-6d17-4daa-ac22-d97d3c66738d

Oxford, Bodleian Library, Opp. Add. $4^{\circ}$ 147c: http://digital.bodleian.ox.ac.uk/inquire/p/263b0aade1ad-4364-9e2e-39d981565b95

Oxford, Bodleian Library, Opp. Add. $4^{\circ}$ 148: http://digital.bodleian.ox.ac.uk/inquire/p/14ce4f24a9a2-431c-984b-535fc80e678f

Oxford, Bodleian Library, Opp. Add. $8^{\circ}$ 7: http://digital.bodleian.ox.ac.uk/inquire/p/b9c98a54100f-4068-a905-81f572fe9c25 
